Esta revista forma parte del acervo de la Biblioteca Jurídica Virtual del Instituto de Investigaciones Jurídicas de la UNAM www.juridicas.unam.mx

\title{
¿EL FIN DEL TRABAJO O EL TRABAJO \\ COMO FIN? PROCESO CONSTITUYENTE Y REFORMAS \\ LABORALES EN EL ECUADOR DE LA “REVOLUCIÓN \\ CIUDADANA" (2007-2013)*
}

THE END OF WORK OR WORK AS END?

CONSTITUTIONAL PROCESS AND LABOR REFORMS IN

ECUADOR'S “CITIZENS’ REVOLUTION” (2007-2013)

LA FIN DU TRAVAIL OU LE TRAVAIL COMME FIN?

PROCESSUS CONSTITUANT ET REFORMES

DU TRAVAIL DANS L'ÉQUATEUR DE LA “REVOLUTION

CITOYENNE” (2007-2013)

Pierre GAUSSENS ${ }^{* *}$

Resumen: La llegada al poder del gobierno de Alianza País en Ecuador se caracteriza por un proceso constituyente en 2008 que declara la abolición de las formas precarias de contratación laboral. Sin embargo, las políticas económicas tomadas en los años siguientes en materia de trabajo, al fomentar la productividad y legalizar el despido injustificado, vulneran las solidaridades obreras y desmienten en los hechos el discurso oficial sobre el fin del trabajo precario.

Palabras clave: Alianza País, reformas laborales, movimiento obrero-sindical, conflictividad.

ABSTRACT: The coming to power of Alianza País government in Ecuador is characterized by a constitutional process in 2008 that declares the abolition of precarious forms of hiring. However, economic policies taken in the following years in labor matters, to greater productivity and

* Recibido el 4 de enero de 2016 y aceptado para su publicación el 1o. de abril de 2016.

** Candidato a doctor en sociología en la UNAM; facilitador en la Universidad de los Pueblos del Sur-Estado de Guerrero (pierre.gaussens@gmail.com). 
Esta revista forma parte del acervo de la Biblioteca Jurídica Virtual del Instituto de Investigaciones Jurídicas de la UNAM

legalize unjustified dismissal, undermine the workers' solidarities and indeed contradict the official discourse about the end of precarious work.

Key Words: Alianza País, labor reforms, labor-union movement, conflict.

RÉSUMÉ: L'arrivée au pouvoir du gouvernement d'Alianza País en Equateur se caractérise par un processus constituant en 2008 qui déclare l'abolition des formes précaires d'embauche. Cependant, les politiques économiques prises en matière de travail durant les années suivantes, en cherchant plus de compétitivité et en légalisant le licenciement injustifié, portent atteinte aux solidarités ouvrières et démentent de fait le discours officiel sur la fin du travail précaire.

Mots-clés: Alianza País, réformes du travail, mouvement ouvrier syndical, conflits.

SumAriO: I. Introducción. II. El proceso constituyente de 2008. III. Politicas económicas y salariales. IV. Políticas laborales posconstituyente. V. Conflictividad laboral. VI. Conclusión. VII. Bibliografía.

El alza del precio del trabajo se mueve siempre dentro de límites que no sólo dejan intangibles las bases del sistema capitalista, sino que además garantizan su reproducción en una escala cada vez más alta.

Carlos MARX

\section{INTRODUCCIÓN}

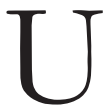
n "elemento del poder del Estado de fundamental importancia para el funcionamiento del capitalismo histórico ha sido el derecho legal de los Estados a determinar las normas que rigen las relaciones sociales de producción dentro de su jurisdicción territorial". ${ }^{1}$

En este sentido, el estado del derecho laboral expresa en todo momento el estado de las relaciones de fuerza, tanto entre el capital y el trabajo, como entre el Estado y el movimiento obrero-sindical. A partir del estudio de la

\footnotetext{
1 Wallerstein, Immanuel, El capitalismo histórico, Madrid, Siglo XXI, 1988, p. 40.
} 
Esta revista forma parte del acervo de la Biblioteca Jurídica Virtual del Instituto de Investigaciones Jurídicas de la UNAM

legislación fabril inglesa por Marx en El Capital, la demostración sobre esta "fundamental importancia" del derecho social para las clases dominantes radica en que todo cambio en los dispositivos legales afectan necesariamente las capacidades de organización y movilización de las clases, y grupos subalternos, en sus condiciones de vida como agentes proletarizados.

En América Latina, a lo largo del año 2000, la llegada al poder de una serie de nuevos gobiernos, con la elección del gobierno de Chávez en Venezuela (1998), Lula en Brasil (2001), Kirchner en Argentina (2003), Morales en Bolivia (2005), Correa en Ecuador (2006), Ortega en Nicaragua (2006) o Mujica en Uruguay (2009), representó un giro a la izquierda en el panorama político regional, con implicaciones de un cambio para el Estado del derecho social. Este giro tiene raíces históricas en las tres décadas anteriores, marcadas por la política exterior estadounidense, como las dictaduras militares y la imposición del neoliberalismo. En este contexto pos-1968, son entonces las resistencias populares a esta imposición, como en el caso de las luchas de los obreros desocupados en el Cono Sur, las que generan las condiciones políticas propicias para la llegada al poder de nuevos gobiernos que, bajo la presión de los movimientos sociales, ahora rechazan la aplicación del Consenso de Washington. En este sentido, el giro a la izquierda del año 2000 debe entenderse en términos históricos como una consecuencia indirecta, una especie de "daño colateral" de las resistencias populares al autoritarismo neoliberal.

Ahora bien, en su oposición formal a las políticas neoliberales y su presentación de izquierda estos nuevos gobiernos, a menudo fueron vistos como los defensores de los intereses del trabajo frente al capital, inclusive desde las mismas organizaciones sindicales. Los discursos oficiales y los análisis dominantes derivados de ellos — construidos en torno a los tópicos del socialismo del siglo XXI y del posneoliberalismo— no dejaron de presentar a este giro a la izquierda como una supuesta "revancha histórica" para las clases trabajadoras, mediante anuncios triunfales sobre el fin del trabajo precario y la flexibilización laboral. En Ecuador, es así como en 2006, el primer programa electoral del partido Alianza País rezaba lo siguiente: "las ganancias del capital no pueden conseguirse en base al hambre y la miseria del trabajo"; "estando en contra de la depredación de las relaciones laborales, entendida

2 Alianza País, Plan de gobierno del Movimiento PAÍS 2007-2011. Un primer gran paso para la transformación radical del Ecuador, Quito, 2006, p. 26. 
como flexibilización laboral, vamos a establecer... control sobre las excesivas utilidades/rentabilidades empresariales que generan procesos perversos de acumulación de riqueza individual y desigualdad social"; "3 "daremos la vuelta a la página. No más neoliberalismo, ni ajuste estructural, ni sumisión al FMI y Banco Mundial. No nos interesan los comentarios y opiniones de Wall Street para asumirlos en nuestras políticas"; " "nuestro gobierno no será un socio de las empresas transnacionales en contra del pueblo ecuatoriano." 5

No obstante, los gobiernos latinoamericanos del giro a la izquierda, más allá del triunfalismo de los discursos dominantes así como de las especificidades que pueden distinguirlos, sobre todo comparten un rasgo central que radica en la cooptación simbólica, desde sus respectivas agendas políticas, de las demandas formuladas históricamente por los movimientos sociales, con el fin de poder metabolizar la impronta subalterna dentro de las leyes del Estado y del mercado. Este proceso corresponde con amplias reformas legales, pero en el caso de ciertos Estados como en Venezuela, Bolivia y Ecuador, implica también procesos constituyentes cuyas innovaciones jurídicas integran los ejes articuladores de las luchas populares al mismo tiempo que transforman la institucionalidad del Estado. En Ecuador, el proceso de la autollamada "revolución ciudadana”, dirigido por el gobierno de Alianza País (en adelante AP), auspicia la adopción en 2008 de una Constitución que establece la abolición de las formas precarias de contratación laboral, incluyendo la intermediación y la tercerización (outsourcing), además de otras innovaciones como son el régimen económico del buen vivir, los derechos de la naturaleza, la plurinacionalidad o la soberanía alimentaria, entre otras.

En este punto, las apariencias propias de la formalidad que da fuerza al derecho, así como las de un discurso oficial que promueven sus innovaciones, no dejan de engañar las percepciones sobre el "proceso de cambio", en Ecuador. Para la nueva fracción de la clase dominante en el poder con el gobierno de AP, lo importante de la integración al Estado de las demandas subalternas no radica en el alcance del cambio que ésta puede generar (o no), sino en la producción de una imagen del cambio. En este sentido, el proceso de la "revolución ciudadana" en Ecuador se asemeja a una revolución conservadora (Bourdieu) o revolución pasiva (Gramsci), en la medida en que busca

\footnotetext{
3 Ibidem, p. 29.

4 Ibidem, p. 44.

5 Ibidem, p. 46.
} 
Esta revista forma parte del acervo de la Biblioteca Jurídica Virtual del Instituto de Investigaciones Jurídicas de la UNAM

poner en práctica el principio de Lampedusa al "cambiarlo todo (o fingir que lo cambian) con el fin de que nada cambie (aunque parezca que sî). Este procedimiento es extremadamente engañoso. El primer problema es inventar el cambio... el segundo es engañar a una gran parte del bando del que se forma parte. El tercero es engañar a los oponentes". ${ }^{6}$ Aquí, el primer problema, el de la invención del cambio, es resuelto por la dimensión innovadora (en términos jurídicos) de la Constitución de 2008. El segundo problema provoca tensiones en el interior de AP a lo largo del proceso constituyente, pero sin que éstas pongan en riesgo la dinámica ascendente de la fracción de clase que representa el nuevo partido. En cambio, el tercer problema se refiere directamente a las políticas de gobierno, en relación con los movimientos sociales y en particular con el movimiento obrero-sindical.

Por tanto, revisemos ahora la evolución de las medidas del gobierno de AP en materias laborales, como normas secundarias de aplicación de una Constitución vanguardista para la jurisprudencia en derecho social. En efecto, la manera de persuadir que la ausencia real de cambio signifique la existencia ficticia del mismo, precisamente, puede tomar el pretexto de la explotación, "de la ecología o del multiculturalismo... [y] es muy difícil para los movimientos sociales no dejarse llevar por la corriente al paso del tiempo, en especial si por este medio pueden obtener parte de sus objetivos inmediatos". 7 Así, sólo el análisis crítico sobre las reformas laborales del gobierno de AP nos permitirá evaluar en qué medida éste representa realmente los intereses del capital, cómo se plasman las nuevas estrategias de dominación para garantizar un control del Estado sobre la fuerza de trabajo, y cuál es el escenario que estas estrategias construyen para el movimiento obrero-sindical en Ecuador.

\section{EL PROCESO CONSTITUYENTE DE 2008}

Sin querer profundizar en la historia del sindicalismo en Ecuador, ${ }^{8}$ no obstante, cabe recalcar que una de las causas profundas del estado general de debilitamiento del movimiento obrero-sindical en el país, a lo largo de los años

6 Wallerstein, Immanuel, Utopística o las opciones históricas del siglo XXI, México, UNAM-Siglo XXI, 1998 , p. 85.

7 Ibidem, p. 86.

8 Ibarra, Hernán, "Los estudios sobre la historia de la clase trabajadora en el Ecuador", Ecuador Debate, Quito, núm. 72, diciembre de 2007, pp. 61-80. 
ochenta y noventa, se encuentra en la implementación de políticas de flexibilización laboral por parte de los sucesivos gobiernos en turno. En consecuencia, en 2008 la conciencia sobre las causas de su propia debilidad conduce a las organizaciones sindicales a luchar ante la Asamblea Nacional Constituyente (ANC) por el final de la intermediación y la tercerización laboral, presentándolo como su principal demanda.

$\mathrm{Al}$ respecto, si bien el gobierno de $\mathrm{AP}$, con su orientación desarrollista de tipo keynesiano, presenta un interés objetivo en mayores niveles de estabilidad laboral para los sectores sociales, objetos del vasto proceso de proletarización que sus políticas económicas buscan orquestar (con el fin de sostener la demanda agregada del mercado interno), al mismo tiempo, el final de toda intermediación laboral equivaldría a un aumento generalizado de los costos de la fuerza de trabajo y, por tanto, a una reducción de las cuotas de plusvalía que a su vez desincentivaría los niveles de inversión productiva del capital. Frente a tal dilema, el pragmatismo de la nueva fracción de la clase dominante ahora en el poder llega a una solución conciliadora, tanto para los intereses de su política económica, como para los de la acumulación del capital. Por un lado, la Constitución de 2008 establece entonces la abolición formal de todas las formas precarias de contratación laboral:

Artículo 327. La relación laboral entre personas trabajadoras y empleadoras será bilateral y directa. Se prohíbe toda forma de precarización, como la intermediación laboral y la tercerización en las actividades propias y habituales de la empresa o persona empleadora, la contratación laboral por horas, o cualquiera otra que afecte los derechos de las personas trabajadoras en forma individual o colectiva.

Sin embargo, por otro lado es garantizada la reproducción real de la tercerización laboral, dado que si ésta es abolida "en las actividades propias y habituales de la empresa", es de entender que sigue siendo permitida en las actividades "inhabituales" de la empresa. Esta interpretación sobre la ambigüedad de la fórmula constitucional es confirmada por la adopción de un mandato constituyente, ${ }^{9}$ conocido como Mandato 08 o mandato laboral, el cual estipula:

9 Los 21 mandatos constituyentes emitidos por la ANC, en 2008, constituyen una figura jurídica controversial, de jerarquía constitucional, mediante la cual dicha asamblea cumple con una función legislativa (dejada vacante por la cesación de actividades del ex Congreso Nacional) sobre materias de coyuntura política. 
Esta revista forma parte del acervo de la Biblioteca Jurídica Virtual del Instituto de Investigaciones Jurídicas de la UNAM

Artículo 3o. Se podrán celebrar contratos con personas naturales o jurídicas autorizadas como prestadores de actividades complementarias por el Ministerio de Trabajo y Empleo, cuyo objeto exclusivo sea la realización de actividades complementarias de: vigilancia, seguridad, alimentación, mensajería y limpieza, ajenas a las labores propias o habituales del proceso productivo de la usuaria.

Artículo 4o. En los contratos a que se refiere el artículo anterior, la relación laboral operará entre los prestadores de actividades complementarias y el personal por esta contratado en los términos de la ley, sin perjuicio de la responsabilidad solidaria de la persona en cuyo provecho se preste el servicio (énfasis nuestro en cursivas).

Éste es el compromiso encontrado por el gobierno de AP y aquí es donde radica el truco legal: el proceso constituyente no elimina todas, sino algunas formas precarias de contratación laboral, como son la intermediación genérica y el contrato por horas, al mismo tiempo que mantiene vigente la tercerización laboral (como forma sectorial de intermediación), la cual ahora es escondida bajo el tecnicismo de "prestación de actividades complementarias" y es circunscrita a los sectores de actividad enumerados en el artículo 4o. del Mandato. Por tanto, mientras que el discurso oficial no deja de ostentar el fin anunciado de la precariedad en el trabajo, la realidad de la explotación laboral no cambia para una parte importante de la fuerza de trabajo. Así es cómo el simple disfraz de un jargón técnico-legal puede permitir la conservación de los intereses empresariales, garantizar la reproducción de la flexibilización del mercado laboral y, a su vez, mantener las condiciones estructurales que impiden la formación de las solidaridades obreras y generan un estado general de debilidad para el conjunto del movimiento obrero-sindical.

Con el proceso constituyente, en 2008, “aparecen nuevas categorías que reemplazan a las existentes, como los servicios técnicos especializados, las actividades complementarias y la jornada parcial de trabajo. Por lo tanto, la inquietud resultante es si ¿realmente desapareció la precarización del trabajo? Ya que a pesar de la disparidad en la información, se calcula que hasta antes de la promulgación del Mandato 08 en el país hubo más de un millón de trabajadores en esa condición. Luego, según cifras oficiales, ingresaron en relación directa de trabajo aproximadamente 200.000 a las entidades y empresas públicas, así como a las empresas privadas; es decir, apenas un $20 \%$. Es de suponerse que el 80\% restante se quedó en esa condición o fueron despedi- 
dos, como en efecto sucedió un año después con un significativo número de trabajadores del sector público y privado". ${ }^{10}$

Es más, el mandato laboral no sólo regula la tercerización de las actividades de trabajo, también implica la violación sistemática de los principios de intangibilidad e irrenunciabilidad del derecho laboral y, en particular, de los contratos colectivos de los trabajadores del Estado, "en contra de las prebendas laborales excesivas de determinados y minúsculos sectores... [y por] el establecimiento de límites mínimos y máximos a los salarios públicos". ${ }^{11}$ En este sentido, la tercera disposición transitoria de dicho Mandato establece que "las cláusulas de los contratos colectivos que no se ajusten a los parámetros a los que se refiere esta disposición transitoria y que contengan privilegios y beneficios desmedidos y exagerados que atentan contra el interés general, son nulas de pleno derecho".

En resumen:

...la ANC en el supuesto afán de acabar con las inequidades y desigualdades remunerativas en el sector público, mediante los mandatos constituyentes 02, 04 y 08, desconoció principios fundamentales del derecho del trabajo... Desconocimiento que afecta irremediablemente a los derechos de organización, contratación colectiva, huelga y estabilidad laboral... La estrategia por lo tanto fue quitar los derechos a los pocos trabajadores con acceso a ellos, para que nadie los tenga. ¡Peculiar forma de terminar con la inequidad! $!^{12}$

Además, y de forma paralela, la figura de la huelga solidaria, suprimida por las reformas de la "larga y triste noche neoliberal", no es reintroducida por la ANC. Al contrario, apoyándose en el artículo 158 del Código Penal que tipifica la paralización de los servicios públicos como delito de sabotaje y terrorismo, la Constitución de 2008 prohíbe de facto el derecho a la huelga para los trabajadores del Estado, a través de un articulado que raya en lo ridículo:

Artículo 326 numeral 14. Se reconocerá el derecho de las personas trabajadoras y sus organizaciones sindicales a la huelga.

10 Cano, Diego, "Regresión laboral en Ecuador y sus consecuencias: gobierno de Rafael Correa", ¿Estado constitucional de derechos? Informe sobre derechos humanos. Ecuador 2009, Quito, Universidad Andina Simón Bolívar, 2010, p. 299.

11 Alianza País, op. cit., p. 29.

12 Cano, Diego, "Regresión laboral...”, op. cit., p. 298. 
Artículo 326 numeral 15. Se prohíbe la paralización de los servicios públicos de salud y saneamiento ambiental, educación, justicia, bomberos, seguridad social, energía eléctrica, agua potable y alcantarillado, producción hidrocarburífera, procesamiento, transporte y distribución de combustibles, transportación pública, correos y telecomunicaciones. La ley establecerá límites que aseguren el funcionamiento de dichos servicios.

Finalmente, el proceso constituyente auspiciado por el gobierno de AP, lejos de terminar con la precariedad laboral y fomentar la defensa de los derechos y la organización del trabajo, desemboca en una Constitución cuyo artículo 326 limita la libertad sindical, mediante un derecho de injerencia del Estado en la creación de las organizaciones obreras (numeral 8); reproduce la desigualdad de un tratamiento legal que segrega los trabajadores del Estado con los del sector privado (numeral 16), mediante el mantenimiento de dos regímenes laborales diferenciados (leyes de carrera administrativa para los primeros, Código del Trabajo para los segundos); y restringe los derechos de contratación colectiva (numeral 13).

\section{POLÍTICAS ECONÓMICAS Y SALARIALES}

En general, la política económica del gobierno de AP se inscribe en el marco del keynesianismo, es decir, en una mayor intervención del Estado dentro del mercado interno cuyo voluntarismo busca aumentar la cuota media de ganancia de la producción vía el consumo de sus mercancías (demanda agregada). A su vez, la activación de este consumo pasa por un proceso de mayor proletarización de las clases trabajadoras, mediante una compra mayor de su fuerza de trabajo por el capital y el pago a cambio de salarios más altos. En este sentido, si la formación bruta de capital fijo (infraestructura) permite la generación de trabajo asalariado para un importante contingente de obreros, esta generación por sí sola no basta para garantizar niveles suficientes de consumo.

Es por esta razón que las grandes obras del keynesianismo de la "revolución ciudadana" se acompañan de políticas salariales tendientes al fortalecimiento de los niveles remunerativos, pues se trata de garantizar el poder adquisitivo de los sectores en vía de proletarización. Por tanto, desde que asume la dirección del Estado, el gobierno de AP decreta alzas sistemáticas del Salario Básico Unificado (SBU) para cada año entrante, pasando éste de 170 dólares mensuales en 2007, a 318 dólares en 2013. 
Esta revista forma parte del acervo de la Biblioteca Jurídica Virtual del Instituto de Investigaciones Jurídicas de la UNAM

\section{FIGURA 1}

\section{SALARIO BÁSICO UNIFICADO (2007-2013) ${ }^{13}$}

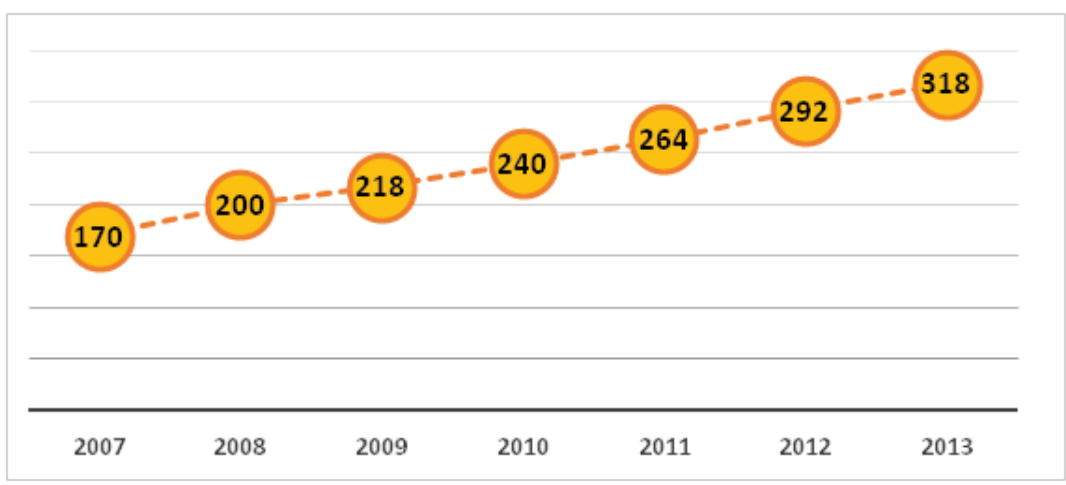

La política salarial del gobierno de AP, basada en el aumento constante del SBU, tiene como objetivo declarado la recuperación de una capacidad general de consumo (poder adquisitivo) y, como objetivo implícito, constituir a los estratos medios beneficiados como pilares políticos del nuevo régimen, así como forzar de manera paulatina la integración de las clases trabajadoras a las reglas del mercado. Mediante la transformación no de las condiciones de producción, sino de las recompensas de la participación en esta producción, se trata, a la postre, de metabolizar la impronta subalterna dentro del consumo mercantil.

Por su lado, las empresas que en un principio ven su cuota de plusvalía vulnerada por la presión hacia el alza de los costos laborales de producción, pueden recuperar parcialmente esta cuota a través de otra, la cuota de ganancia, ${ }^{14}$

13 Ministerio Coordinador de Desarrollo Social, Sistema integrado de indicadores sociales del Ecuador, Quito, 2013.

14 En El Capital (tomo III, sección primera), Marx distingue ambas cuotas: mientras que la cuota de ganancia se realiza en el proceso de circulación del mercado y expresa la diferencia entre el costo global de producción (o capital total desembolsado) y el precio de venta de la mercancía producida, constituyendo así un mecanismo simple, por ejemplo, característico del capital comercial; en cambio, la cuota de plusvalía se realiza dentro del proceso de producción mismo, reflejando el grado de explotación de la fuerza de trabajo por el capital, y refiriéndose a la diferencia entre el precio de esta fuerza (transfigurado en salario) y el valor que realmente crea su función, como trabajo vivo. Así, la cuota de plusvalía es la diferencia entre el valor pagado del trabajo socialmente necesario y el valor no retribuido del trabajo excedente, siendo la principal differentia specifica (junto con la producción de mercancías) del capitalismo 
Esta revista forma parte del acervo de la Biblioteca Jurídica Virtual del Instituto de Investigaciones Jurídicas de la UNAM

que es generada por la venta de las mercancías producidas, compradas gracias al alza salarial, y que es ampliada a mediano plazo con la venta de un volumen aún mayor de mercancías, adquiridas gracias a la constancia del alza (sin contar con una tendencia inflacionaria hacia un aumento general en los precios de estas mercancías). En efecto, "una mayor remuneración a los trabajadores podía en ciertas circunstancias retornar a los acumuladores de capital como ganancia diferida, a través del mayor poder adquisitivo global en la economía". ${ }^{15}$

En el Ecuador de la "revolución ciudadana" el aumento constante del SBU, como expresión de una clara voluntad política por parte del gobierno de AP, llega a presentar de manera relativa el efecto multiplicador (anhelado por Keynes) sobre el incremento de la demanda agregada. En un primer momento, este "efecto dómino" se expresa en el alza del nivel promedio de remuneración para los diferentes sectores laborales del mercado interno, divididos oficialmente entre el sector formal, informal y doméstico, el primero pasa de un salario promedio de 485 a otro de 613 dólares mensuales, el segundo de 218 a 301 dólares, y el tercero de 151 a 265 dólares, entre 2007 y 2012.

\section{FIGURA 2}

NIVEL PROMEDIO DE REMUNERACIÓN

POR SECTOR LABORAL $(2007-2012)^{16}$

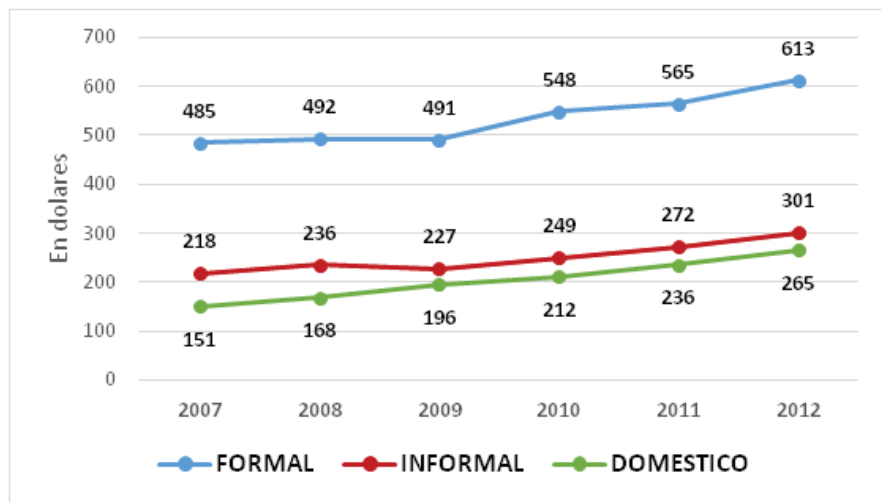

para la generación de valor, mediante la imposición del régimen asalariado (capital variable) y la industrialización de los medios de producción (capital constante).

15 Wallerstein, Immanuel, El capitalismo..., cit., p. 51.

16 Secretaría Nacional de Planificación y Desarrollo, Buen vivir. Plan nacional 2013-2017, Quito, 2013, p. 279. 
Esta revista forma parte del acervo de la Biblioteca Jurídica Virtual del Instituto de Investigaciones Jurídicas de la UNAM

No obstante, cabe recordar que un salario nominal no es un salario real, por lo que esta tendencia general de alza salarial, en un segundo momento, debe ser contrastada con la evolución de una canasta básica cuyo costo necesariamente aumenta en el mismo lapso de tiempo. En efecto, el mayor "enemigo" de todo keynesianismo es la inflación, pues con ésta, el esfuerzo político tendiente a garantizar las condiciones para un mayor consumo de mercancías sería vano (como en el caso, por ejemplo, de la "revolución bolivariana" en Venezuela). En Ecuador, la conjugación del alza salarial generalizada con una tasa promedio de inflación anual (4,7\%) moderada (por la dolarización) permite al keynesianismo de la "revolución ciudadana" alcanzar su objetivo principal: el aumento (aunque desigual) de los salarios reales, que significa el incremento real de los niveles de consumo, ambos reflejados por la evolución positiva del porcentaje con el que el ingreso familiar promedio cubre el costo de la canasta básica, el cual pasa de un 70\% en 2007 a un 99\% para el 2013.

\section{FIGURA 3}

\section{COBERTURA DE LA CANASTA BÁSICA POR EL INGRESO}

FAMILIAR PROMEDIO (2007-2013) ${ }^{17}$

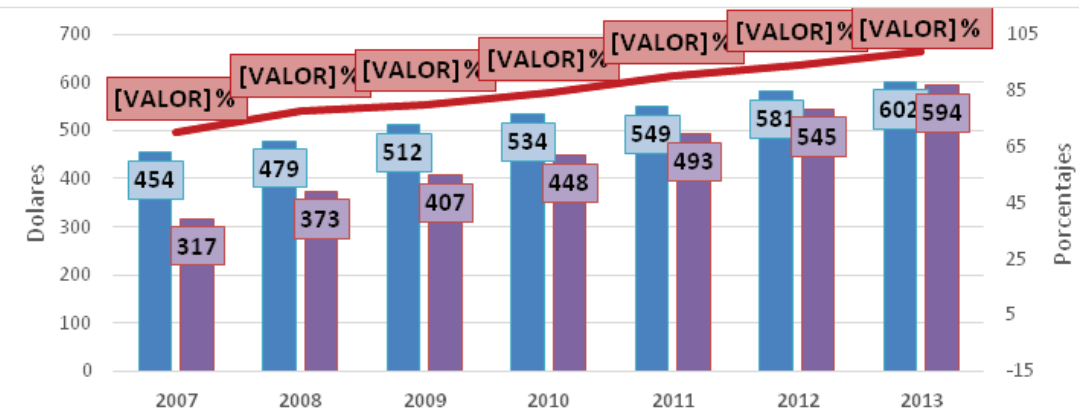

Canasta Basica

Ingreso Familiar

$\%$ de Cobertura de la Canasta Basica

Bajo la "revolución ciudadana", son cada vez más las unidades domésticas cuyo ingreso cubre el costo de la canasta básica, pasando de un $40 \%$ al $46 \%$ del total nacional entre 2007 y 2012, cumpliendo así con el requisito previo a

\footnotetext{
17 Ministerio de Coordinación de la Política Económica, Ecuador económico, Quito, 2013, p. 3.
} 
Esta revista forma parte del acervo de la Biblioteca Jurídica Virtual del Instituto de Investigaciones Jurídicas de la UNAM

la entrada al pleno consumo. A su vez, en el mismo periodo, la representación en la Población Económicamente Activa (PEA) de las unidades domésticas proletarizadas (empleo) aumenta del 35\% al 43\%, mientras que la de las unidades subproletarizadas (subempleo) se reduce del 59\% al 51\%, al mismo tiempo que el desempleo del 5\% al 4\% (figura 4). En suma, el principal resultado de las políticas keynesianas del gobierno de AP, en materias económicas y salariales, consiste en un proceso general de proletarización de la fuerza de trabajo sin precedentes en el país.

\section{FIGURA 4}

ESTRUCTURA DE LA PEA (2007-2012) ${ }^{18}$

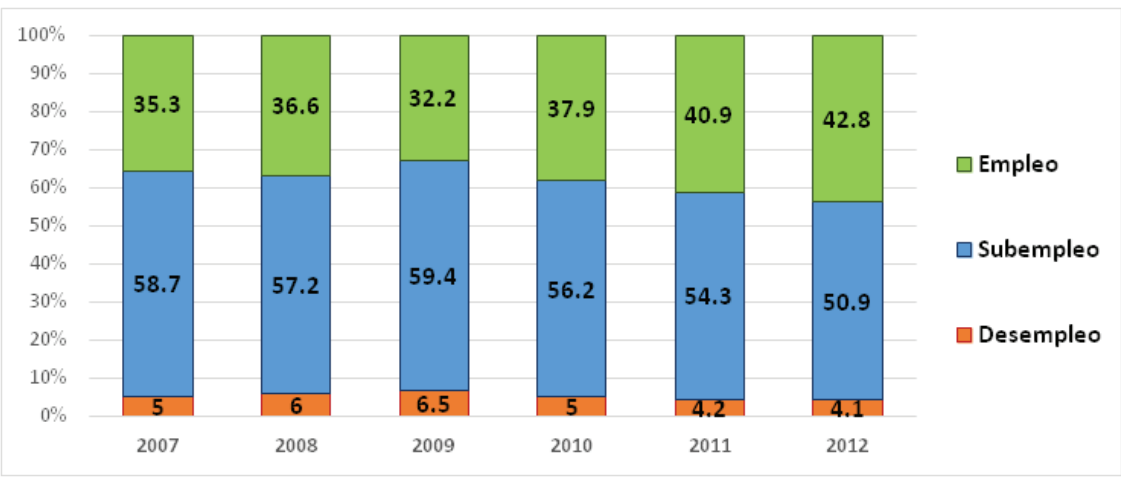

Contra toda lectura formalista, que suele oponer de manera maniquea poder constituyente y poder constituido, ésta tiende a idealizar el proceso constituyente $y$, al mismo tiempo, a satanizar la praxis del gobierno de los años siguientes sobre los tópicos de la "revolución traicionada"; en cambio, hemos evidenciado que la Constitución de 2008, en sus mismos contenidos, ya refleja una serie de profundas contradicciones que expresan una coyuntura marcada por una relación de fuerza favorable al gobierno y a la fracción ascendente de la clase dominante que éste representa. Al revés, "la subsidiaridad de los movimientos sociales a la lógica política que se desarrollaba en ese entonces

18 Secretaría Nacional de Planificación y Desarrollo, op. cit., p. 276. 
Esta revista forma parte del acervo de la Biblioteca Jurídica Virtual del Instituto de Investigaciones Jurídicas de la UNAM

no ayudó a la reconstrucción y empoderamiento de éstos, dejándolos en una posición subalterna respecto a la política institucional". ${ }^{19}$

En el proceso constituyente de 2008, las plataformas de demandas construidas desde los movimientos sociales y llevadas a la ANC por sus organizaciones, o son tomadas en cuenta e integradas parcialmente al texto constitucional, o bien se quedan como declaraciones de principio, o simplemente son descartadas por no encajar en el escenario keynesiano que las políticas económicas del nuevo gobierno buscan construir. Así, el relativo grado de apertura del proceso constituyente, en el periodo (2007-2009) coincide con los niveles altos de negociación (33.2\%) frente a los posibles conflictos, así como de desenlace positivo $(30.2 \%)$ para los mismos, y con un nivel bajo de rechazo $(6.5 \%)$ ante las demandas formuladas hacia el gobierno. Sin embargo, este primer momento de integración formal y parcial de las demandas sociales en el Estado reformado contrasta con un segundo momento, en el periodo (2010-2012) cuando la tasa de desenlace positivo de los conflictos cae de 8 puntos a un $22.3 \%$, el aplazamiento de resolución sube de 4 puntos a un $14.7 \%$ y, sobre todo, el nivel de rechazo de AP hacia los conflictos sociales casi triplica respecto de su primer periodo de gobierno, para alcanzar un $18.1 \%$ del total.

\section{CUADRO 1}

DESENLACE DE LOS CONFLICTOS SOCIALES (2007-2009/2010-2012)20

\begin{tabular}{|l|c|c|}
\hline \multicolumn{1}{|c|}{ Desenlace } & 2007-2009 & 2010-2012 \\
\hline Aplazamiento de la resolución & 10.8 & 14.7 \\
\hline Negociación & 33.2 & 29.9 \\
\hline No negociación & 11.9 & 10.1 \\
\hline Positivo & 30.2 & 22.3 \\
\hline Rechazo & 6.5 & 18.1 \\
\hline Represión & 5 & 4.8 \\
\hline
\end{tabular}

19 Machado, Decio, “¿Una nueva etapa de los movimientos sociales en Ecuador?”, La Tendencia, Quito, núm. 13, abril-mayo de 2012, p. 16.

20 Sánchez, Paola, "Resistencia, consenso y disputa: reflexión sobre el conflicto social en el Ecuador (1990-2012)”, ¿A quién le importa los guayacanes?, Quito, Instituto de Estudios Ecuatorianos, 2013, p. 65. 
Esta revista forma parte del acervo de la Biblioteca Jurídica Virtual del Instituto de Investigaciones Jurídicas de la UNAM

Esta evolución negativa en el desenlace de los conflictos sociales ilustra los límites conservacionistas del "proceso de cambio" en Ecuador entendido como revolución conservadora. O cuando la integración formal de las demandas sociales en el proceso constituyente deja lugar a su desintegración real en las políticas de gobierno. En este sentido, las políticas laborales del gobierno de AP en el periodo posconstituyente son ejemplares.

Combinan varios elementos: la fragmentación de los trabajadores en trabajadores públicos y privados, para evitar su constitución en sujetos; el enfrentamiento de los trabajadores públicos de cara a los "ciudadanos", debilitando sus posibilidades de organización y de acción reivindicativa; pero también dando satisfacción a ciertas aspiraciones de los trabajadores, represadas en el periodo anterior, como mejores salarios y posibilidades organizativas (para los trabajadores del sector privado), es decir, desactivando ciertas inconformidades sociales. Al mismo tiempo, se mantienen espacios de trabajo flexibilizado, en el sector privado disfrazado ahora de encadenamientos productivos y de negocios inclusivos, y en el sector público disfrazado de contratos por tiempo fijo. ${ }^{21}$

En general, la coyuntura propia a la llegada al poder de AP se caracteriza por un estado general de debilitamiento del movimiento obrero-sindical, sobre todo en el sector privado, al respecto del cual el nuevo gobierno se restringe en fomentar la participación accionarial de los trabajadores en el capital de la empresa, bajo el lema de "democratizar los medios de producción" y mediante exoneraciones fiscales para las empresas (previstas en el Código de la Producción de 2010). Para la fracción de la clase dominante en ascensión, la potencial peligrosidad del sindicalismo no se ubica en el sector privado, sino en el sector estatal, ya que el estado de debilidad del movimiento obrero-sindical en Ecuador, a mediados de 2000, tiene como notable excepción a las organizaciones de los trabajadores del Estado.

Entonces, apoyadas en una Constitución de 2008 que restringe el derecho a la huelga, y en un mandato laboral que abre paso a la revisión de los esquemas de contratación colectiva, las primeras políticas laborales del gobierno de AP se destinan a la disposición de una fuerza de trabajo disciplinada

21 Unda, Mario, "Modernización del capitalismo y reforma del Estado", El correísmo al desnudo, Quito, Montecristi Vive, 2013, p. 35. 
en por y para el Estado, recolectando "en su beneficio el sentido común generado por el neoliberalismo en contra del Estado, transfiriéndolo a sus trabajadores y a sus organizaciones sindicales... Se construyó la imagen odiosa del burócrata insensible a las necesidades de la gente... [y] los ataques en su contra son fáciles y generan popularidad para el gobierno y para su reforma del Estado". 22

Para tal fin fue promulgado en abril de 2009 el Decreto Ejecutivo 1701, el cual regula el régimen de contratación colectiva en el sector público, reglamenta el despido injustificado, limita los permisos sindicales y la realización de asambleas, así como elimina las contribuciones patronales para la contratación de seguros de salud para los trabajadores. Así, este decreto representa un primer paso en el "adiestramiento" de la fuerza de trabajo del sector estatal, al limitar la capacidad de acción sindical y facilitar la terminación unilateral de la relación laboral. Por ejemplo, la precarización de las condiciones de trabajo en el sector estatal se traduce en una expansión de los contratos civiles por prestación de servicios personales, como contratos de tiempo fijo, no amparados por el Código del Trabajo, que pasan entre 2008 y 2013 de un 25\% a un $49 \%$ del total de "gastos en personal para inversión" del Presupuesto General del Estado (PGE) pagado. 23

Ahora bien, un segundo paso, más firme esta vez, se da con el Decreto Ejecutivo 813 de julio de 2011, puesto que su octavo artículo reforma el Reglamento General de la Ley Orgánica de Servicio Público, al añadirle el siguiente artículo innumerado:

Cesación de funciones por compra de renuncias con indemnización.- Las instituciones del Estado podrán establecer planes de compras de renuncias obligatorias con indemnización... debidamente presupuestados, en virtud de procesos de reestructuración, optimización y racionalización de las mismas... Las servidoras y servidores públicos deberán cumplir obligatoriamente estos procesos aplicados por la administración... La compra de renuncias con indemnización no es aplicable... para los puestos comprendidos dentro de la escala del nivel jerárquico superior (énfasis nuestro en cursivas).

22 Unda, Mario, "Ecuador 2011, el año 5. La coyuntura y el gobierno de la revolución ciudadana”, Observatorio Social de América Latina, Buenos Aires, núm. 31, mayo de 2012, p. 209.

23 Gachet, Francisco y Carrión, Diego, “Ámbito economía”, Balance crítico del gobierno de Rafael Correa, Quito, Universidad Central del Ecuador, 2014, p. 61. 
Esta revista forma parte del acervo de la Biblioteca Jurídica Virtual del Instituto de Investigaciones Jurídicas de la UNAM

Aquí aparece una figura legal sui géneris, la "compra de renuncia obligatoria" (sic), la cual permite a los servicios administrativos del Estado obligar a todo miembro de su personal a renunciar a su puesto de trabajo, sin previo aviso, a cambio de una indemnización. Esta figura, que es altamente arbitraria y coercitiva, se convierte entonces en el mecanismo por excelencia de un proceso general de depuración del conjunto de los cuerpos burocráticos, directamente inspirado en técnicas del management empresarial tendientes a la extracción de mayores cuotas de plusvalía relativa, mediante el fomento a la productividad del trabajo. A su vez, se basa en la evaluación con carácter punitivo del "desempeño" del trabajador del Estado, por lo que expresa el afán (hegeliano) de "racionalización” del país por parte de la nueva clase dominante, de carácter meritocrático, a cuyos puestos de dirección esta medida "no es aplicable".

En este nuevo contexto,

el Ministerio de Relaciones Laborales desarrolla roles que históricamente fueron destinados al sindicalismo. A través de los funcionarios públicos se resuelven conflictos laborales en los cuales la función del Estado no es la defensa de los puestos de empleo, sino que los trabajadores cobren adecuadamente sus indemnizaciones en caso de despido... El menosprecio institucional hacia las organizaciones de trabajadores ha derivado en que la negociación colectiva brille por su ausencia. ${ }^{24}$

Con la "compra de renuncia obligatoria" se abre una vía inédita en el país hacia la legalización del despido injustificado, así como el entierro de la relativa estabilidad de la carrera administrativa.

El resultado es el siguiente:

Los despidos de miles de trabajadores y trabajadoras realizados en el Ecuador a finales de octubre de 2011 y principios de 2012, no sólo revelan la implantación a la fuerza de un modelo de institucionalidad pública necesario para acomodar y adaptar el Estado a los nuevos requerimientos del capital; también, la necesidad de refrescar la militancia pública incondicional al régimen, disfrazada bajo la figura de "recambio generacional", muy en sintonía con las políticas laborales de la llamada "larga noche neoliberal". 25

24 Machado, Decio, op. cit., p. 18.

25 Buitrón, Ricardo y Cano, Diego, "La revolución ciudadana y los trabajadores", Informe sobre derechos humanos. Ecuador 2011, Quito, Universidad Andina Simón Bolívar, 2012, p. 111. 
Esta revista forma parte del acervo de la Biblioteca Jurídica Virtual del Instituto de Investigaciones Jurídicas de la UNAM

En este sentido, el proceso general de depuración de los servicios administrativos busca (más que garantizar atención a los "usuarios") romper con el poder de resistencia de las organizaciones sindicales preexistentes a las políticas del gobierno de AP, al mismo tiempo que los puestos derivados de vacantes de "obligadas renuncias" abren espacios para la constitución de nuevos cuerpos de burócratas, sobre la base de redes clientelares y apoyos selectivos que garanticen, no la competencia profesional de los advenedizos, sino su lealtad hacia quienes fomentaron su acceso. Entonces, “junto a la renovación de las élites políticas y gremiales, está en marcha la creación y consolidación de una capa social muy joven, que por lo tanto podría mantenerse al menos $20 \mathrm{o}$ 25 años en el aparato estatal, ofreciendo una base de respaldo relativamente estable al nuevo régimen". 26

Este "recambio generacional" no es circunscrito a algunas instituciones del Estado, sino más bien es generalizado al entero aparato burocrático. A finales de 2011 "existen despidos masivos en, por lo menos, 83 instituciones del Estado, 73 de ellas corresponden a la Función Ejecutiva, con un total de 9.000 personas despedidas de sus puestos de trabajo". ${ }^{27}$

A estos casos deben sumarse los despidos que se dieron una vez que el Mandato 08 cumplió un año de vigencia, con el que supuestamente terminaba en el país la tercerización e intermediación laboral; porque a partir de abril de 2009 miles de trabajadores de los sectores público y privado, que antes se encontraban en esa condición, fueron despedidos. ${ }^{28}$

Para darnos cuenta del alcance de las purgas, según el Boletín de Ejecución Presupuestaria emitido por el Ministerio de Finanzas en el periodo de enero-marzo de 2012, el PGE del mismo año contempla un monto de $484 \mathrm{mi}-$ llones de dólares (525 en 2013) para financiar compras de renuncias obligatorias, bajo la curiosa denominación de "gastos en personal para la inversión". 29

26 Unda, Mario, “Ecuador 2011...”, op. cit., p. 209.

27 A inicios de 2012 el número pasó a ser de 12.000 trabajadores, según datos estimados por el mismo autor con base en archivos de la Comisión de Derechos de los Trabajadores y Seguridad Social de la Asamblea Nacional, artículos de prensa, reportes directos de trabajadores afectados e información incompleta dada por el Ministerio de Relaciones Laborales.

28 Cano, Diego, Persecución y criminalización de la lucha laboral en el Ecuador. Periodo 2007-2011, Quito, Internacional de Servicios Públicos, 2011, p. 13.

29 Los elevados montos destinados a las "compras de renuncias obligatorias" se explican por el carácter legal excepcional del mecanismo de compra, lo que permite que éstas sean 
Esta revista forma parte del acervo de la Biblioteca Jurídica Virtual del Instituto de Investigaciones Jurídicas de la UNAM

Es decir, casi el 10\% del monto total de inversión previsto en el presupuesto estatal de 2012 es dedicado exclusivamente a financiar despidos de importantes contingentes de funcionarios, nutridos además por opositores políticos y dirigentes sindicales, en todos los sectores administrativos del Estado.

En efecto, "son varios los dirigentes despedidos en el periodo 2007-2011. Hay por lo menos diez casos de persecución y criminalización de la protesta laboral, con acciones incluso de judicialización, en los que se hallan involucrados 48 dirigentes sindicales, gremiales y otros trabajadores". ${ }^{30}$ Por ejemplo, del total de causas ingresadas al sistema judicial en relación con delitos contra la seguridad del Estado, entre noviembre de 2008 y octubre de 2013, un 40\% obedece a razones de "seguridad interior", del cual una mayoría de causas es motivada por "desobediencia en el sector público", ${ }^{31}$ es decir, está vinculada con la resistencia de los trabajadores del Estado ante la depuración de los cuerpos administrativos por la "compra de renuncias obligatorias", "evidenciando que en el país existen hechos de hostigamiento, velados o visibles, que generan temor entre los trabajadores, desestimulan la participación en la actividad sindical de los demás miembros de las organizaciones, fundamentalmente el segmento de jóvenes".32

Finalmente, al mismo tiempo que el gobierno de AP busca debilitar a las organizaciones sindicales más autónomas, inclusive criminalizando su dirección (en un doble sentido), desde el Estado es fomentado un sindicalismo amarillista, con base en el nuevo derecho constitucional de injerencia del Estado en las organizaciones gremiales (artículo 326 numeral 8), a través de la creación ex nibilo de centrales sindicales "amigas", como en el caso de la recientemente creada Confederación de Trabajadores del Sector Público. En este punto, "no puede dejar de llamar la atención cómo un gobierno "progresista" golpea al sindicalismo público, uno de los pocos espacios de organización laboral que habían sobrevivido a la ofensiva neoliberal, y al mismo

financiadas por los ingresos fiscales no permanentes de la renta petrolera, la cual, lejos de beneficiar a la inversión estatal en un "sector social" financiado más bien por los ingresos fiscales permanentes de la recaudación tributaria, sirve en cambio para solventar los planes de reestructuración de las dependencias burocráticas del Estado.

30 Buitrón, Ricardo y Cano, Diego, op. cit., p. 113.

31 Programa Andino de Derechos Humanos, Informe sobre derechos humanos. Ecuador 2009-

2013, Quito, Universidad Andina Simón Bolívar, 2014, p. 97.

32 Cano, Diego, Persecución y criminalización..., cit., pp. 21 у 22. 
tiempo inaugura un tipo de sindicalismo dependiente del Estado, algo que no habíamos tenido hasta ahora". ${ }^{33}$

\section{CONFLICTIVIDAD LABORAL}

El nuevo escenario del mercado interno del trabajo, construido por y desde las políticas laborales del gobierno de AP, se caracteriza por una violación sistemática a los derechos y una precarización de las condiciones de trabajo, ambas apoyadas en un chantaje del discurso oficial acerca del alza promedio de los niveles remunerativos y su cobertura creciente del costo de la canasta básica. El eslogan podría ser entonces: “Trabajar peor para ganar un poco más" o cuando, para garantizar cierta perennidad en la extracción de plusvalía, la elevación del costo de la fuerza de trabajo (salario) busca ser compensada por una mayor exigencia de productividad. Sin embargo, esta última no queda exenta de resistencia por parte de las clases trabajadoras, al contrario, el fomento del gobierno de AP a la "reestructuración, optimización y racionalización" de la producción se acompaña de un incremento generalizado de los conflictos laborales.

Es así como, precisamente a partir de 2007 (año de la llegada al poder de AP), observamos una inversión proporcional en las tendencias de conflictividad, tanto en materias salariales como laborales. En efecto, mientras que por un lado, el nivel promedio de conflictividad salarial (por mayores niveles de remuneración) desciende a partir del gobierno de AP para estabilizarse en un nivel (7\% entre 2007 y 2012) inferior al de los tres periodos anteriores (16\% entre 1990 y 2006); por otro lado, de manera inversa y aún más pronunciada, el nivel promedio de conflictividad laboral (por mejores condiciones de trabajo) no deja de crecer con la "revolución ciudadana", pasando de un 7\% para los tres periodos anteriores a un 25\% entre 2007 y 2012 (figura 5). En el Ecuador de la "revolución ciudadana", combinado entre el sector estatal y privado, el aumento de la conflictividad de carácter laboral, es decir, de las luchas obreras por mejores condiciones de trabajo es tal que llega a representar la mitad (48\%) del nivel total de conflictividad a nivel nacional, entre 2008 y 2013 (figura 6).

33 Unda, Mario, “Ecuador 2011...”, op. cit., p. 209. 
Esta revista forma parte del acervo de la Biblioteca Jurídica Virtual del Instituto de Investigaciones Jurídicas de la UNAM www.juridicas.unam.mx

\section{FIGURA 5}

CONFLICTIVIDAD LABORAL Y CONFLICTIVIDAD SALARIAL POR PERIODO (1990-1996/1997-2001/2002-2006/2007-2009/2010-2012)34

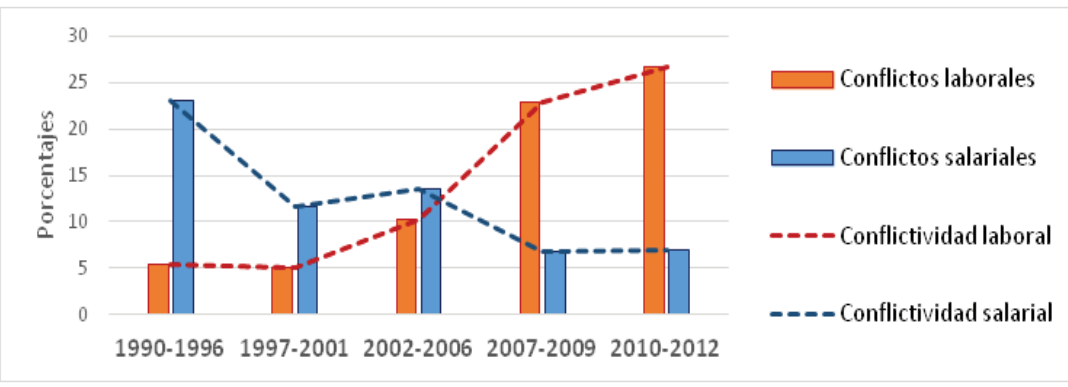

FIGURA 6

TiPOS DE CONFLICTIVIDAD SOCIAL (2008-2013) 35

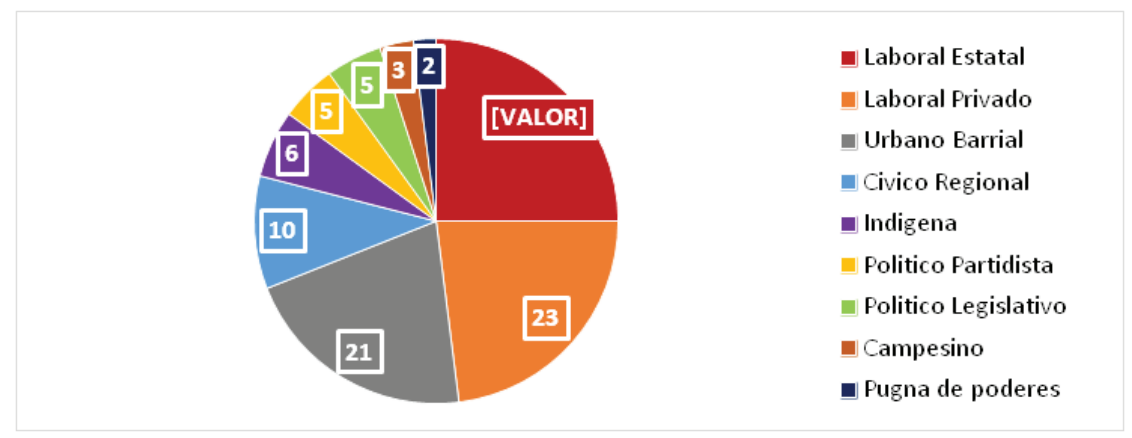

Ahora bien, el debilitamiento activo de la organización sindical por parte del gobierno de AP mediante sus políticas laborales, junto con el alza de la conflictividad laboral en el país, se explica por el hecho de que las clases trabajadores inmersas en este nuevo escenario político y ante la persistencia del antagonismo clasista, ahora orienten sus luchas por fuera de los canales sindicales, al desarrollar formas de organización y movilización innovadoras en

34 Sánchez, Paola, op. cit., p. 34.

35 Programa Andino de Derechos Humanos, op. cit., p. 43. 
Esta revista forma parte del acervo de la Biblioteca Jurídica Virtual del Instituto de Investigaciones Jurídicas de la UNAM

torno a la protesta social, que dejan de obedecer a las lógicas instrumentales de antaño, en torno a la huelga (cuyo nivel nacional baja de un $20 \%$ promedio entre 2002 y 2006 a un 4\% entre 2008 y 2013). ${ }^{36}$ En consecuencia, entre 2008 y 2013, mientras que las clases trabajadoras representan el 26\% de los agentes directos de conflictividad, los sindicatos lo hacen apenas un 5\% (figura 7), los cuales, además, sólo están involucrados en el $20 \%$ de todos los conflictos registrados de carácter laboral. ${ }^{37}$

\section{FIGURA 7}

AgENTES DE LOS CONFLICTOS (2008-2013) ${ }^{38}$

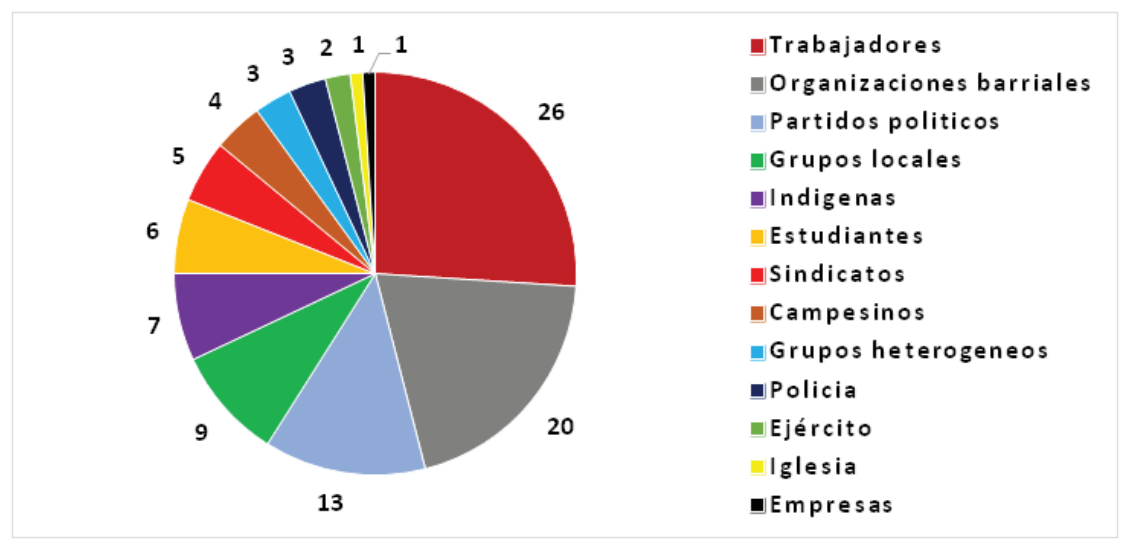

Asimismo, entre 2010 y 2012, los conflictos sostenidos directamente por las clases trabajadoras conocen mayores índices de éxito en su desenlace y relación con el Estado, en comparación con los conflictos intervenidos por los sindicatos, a través de mayores niveles de desenlace positivo y menores tasas de aplazamiento, ${ }^{39}$ lo cual explica que hoy estas mismas clases opten por repertorios de acción por fuera de la intermediación sindical. En suma, las dinámicas recientes de la conflictividad salarial y laboral dan indicios fuertes sobre la evolución de las contradicciones entre capital y trabajo en el Ecua-

\footnotetext{
36 Sánchez, Paola, op. cit., p. 56.

37 Programa Andino de Derechos Humanos, op. cit., p. 44.

38 Idem.

39 Sánchez, Paola, op. cit., p. 69.
} 
Esta revista forma parte del acervo de la Biblioteca Jurídica Virtual del Instituto de Investigaciones Jurídicas de la UNAM

dor de la "revolución ciudadana", las cuales tienden hoy a expresarse más en términos de condiciones laborales, productividad y extracción de plusvalía relativa (so pretexto de competitividad), que en relación con las viejas luchas salariales del movimiento obrero-sindical.

\section{CONCLUSIÓN}

A pesar de la permanencia de las relaciones de producción como principal eje de conflictividad social en el país, expresado en niveles crecientes de conflictos laborales a lo largo de los seis primeros años del gobierno de AP, el proceso de la "revolución ciudadana" en Ecuador ha logrado en los hechos reales desmentir la verdad oficial y las interpretaciones dominantes derivadas de ella. Más allá de su presentación formal de izquierda, el gobierno de AP que lidera dicho proceso, mediante las reformas constitucionales y legales que emprendió en materias económicas y laborales, busca garantizar la reproducción disfrazada de las condiciones estructurales de la precariedad en el trabajo y, por tanto, del debilitamiento activo desde el campo jurídico sobre un sindicalismo que sigue representando una amenaza potencial para la nueva fracción de la clase dominante ahora en el poder, tanto para sus planes de gobierno a corto plazo, como para los procesos generales de acumulación del capital a largo plazo.

En cierta medida, la reducción de los niveles de conflictividad salarial indica que, por más paradójico que pueda parecer, el giro a la izquierda — en Ecuador-, a través de un gobierno de apariencia socialista, ha logrado en apenas seis años lo que las políticas de flexibilización laboral implementadas por los gobiernos abiertamente neoliberales no habían podido hacer, después de dos décadas en el poder, sino sólo parcialmente frente a la resistencia de los movimientos sociales: legitimar la precariedad en el trabajo y legalizar el despido injustificado.

En este escenario político renovado, la creciente precariedad en el trabajo, ya no exclusiva del sector privado, sino extendida al sector estatal por la normalización del despido, sin lugar a duda afecta las capacidades de organización y movilización de las clases trabajadoras, en la medida en que convierte el futuro en algo incierto, frustra toda anticipación sobre el curso del tiempo y, así, contribuye a la pérdida colectiva de ese mínimo de creencia y esperanza 
en el porvenir, que permite rebelarse contra el presente para proyectarse en la búsqueda y construcción de alternativas presentes para un futuro otro.

Sin embargo, con el paso del tiempo para AP en el poder, el presente cada vez más intolerable de las condiciones de trabajo ha provocado el distanciamiento de la mayoría del movimiento obrero-sindical hacia el gobierno de la "revolución ciudadana". Aún de carácter defensivo, las movilizaciones de las organizaciones sindicales en la actual coyuntura se acompañan de otras que ahora buscan, de manera más combativa, resistir los embates de las purgas en el Estado, así como reconstruir los tejidos de la solidaridad obrera en el sector privado, mediante una mayor coordinación de las acciones sindicales, sus agendas y procesos de lucha. En fin, se trata de poder superar las barreras (legales) que siguen dividiendo a los sectores públicos y privados, distanciar los intereses del trabajo calificado del no calificado, y separar a las clases empleadas de los grupos subempleados y desempleados, no con el fin de tener al trabajo como fin, sino con la utopía de seguir haciendo posible en el presente el futuro fin del trabajo.

\section{BIBLIOGRAFÍA}

ALIANZA PAís, Plan de gobierno del Movimiento PAÍS 2007-2011. Un primer gran paso para la transformación radical del Ecuador, Quito, 2006.

Buitrón, Ricardo y CANO, Diego, "La revolución ciudadana y los trabajadores”, Informe sobre derechos humanos. Ecuador 2011, Quito, Universidad Andina Simón Bolívar, 2012.

CANO, Diego, "Regresión laboral en Ecuador y sus consecuencias: gobierno de Rafael Correa", ¿Estado constitucional de derechos? Informe sobre derechos humanos. Ecuador 2009, Quito, Universidad Andina Simón Bolívar, 2010.

—, Persecución y criminalización de la lucha laboral en el Ecuador. Periodo 20072011, Quito, Internacional de Servicios Públicos, 2011.

GACHET, Francisco y CARrión, Diego, “Ámbito economía”, Balance crítico del gobierno de Rafael Correa, Quito, Universidad Central del Ecuador, 2014.

MACHADO, Decio, “¿Una nueva etapa de los movimientos sociales en Ecuador?”, La Tendencia, Quito, núm. 13, abril-mayo de 2012. 
Esta revista forma parte del acervo de la Biblioteca Jurídica Virtual del Instituto de Investigaciones Jurídicas de la UNAM

Ministerio Coordinador de Desarrollo Social, Sistema integrado de indicadores sociales del Ecuador, Quito, 2013.

Ministerio de CoORdinación DE LA POlítica ECONÓMICA, Ecuador económico. Indicadores macroeconómicos, Quito, 2013.

Programa Andino de Derechos Humanos, Informe sobre derechos humanos. Ecuador 2009-2013, Quito, Universidad Andina Simón Bolívar, 2014.

SÁNCHEZ, Paola, "Resistencia, consenso y disputa: reflexión sobre el conflicto social en el Ecuador (1990-2012)", ¿A quién le importa los guayacanes?, Quito, Instituto de Estudios Ecuatorianos, 2013.

Secretaría Nacional de Planificación y Desarrollo, Buen vivir. Plan nacional 2013-2017, Quito, 2013.

UNDA, Mario, "Ecuador 2011, el año 5. La coyuntura y el gobierno de la revolución ciudadana", Observatorio Social de América Latina, Buenos Aires, núm. 31, mayo de 2012.

, "Modernización del capitalismo y reforma del Estado", El correísmo al desnudo, Quito, Montecristi Vive, 2013.

WALLERSTEIN, Immanuel, El capitalismo histórico, Madrid, Siglo XXI, 1988. - Utopistica o las opciones históricas del siglo XXI, México, UNAM-Siglo XXI, 1998. T) 